Fundamental principles of electricity provide a basis for understanding the design and operation of electromyography equipment. An intuitive and quantitative explanation of charge, voltage, current, and impedance provides an introduction to the concepts of resistance, capacitance, and input impedance. These concepts form the basis for discussion of filters, amplifiers, electrodes, digital electronics, stimulators, and patient safety. The monograph assumes no specialized training in engineering or mathematics. The topics are discussed at an introductory level to provide understanding for readers with no electronics background and intuitive insight for more experienced readers.

Key words: electronics • electromyography • electromyography equipment

MUSCLE \& NERVE 14:937-946 1991

\title{
AAEM MINIMONOGRAPH \#36: BASIC CONCEPTS OF ELECTRICITY AND ELECTRONICS IN CLINICAL ELECTROMYOGRAPHY
}

DANIEL T. BARRY, MD, PhD

$W_{\text {hat }}$ is the purpose of electromyography instrumentation? A first thought is that the electromyograph should accurately display the electric signal present at the active electrode. Wrong. Most of the electric signal at the electrode is useless. The electric signals emitted by nerve and muscle are only tiny perturbations superimposed on mountains of noise. The purpose of electromyography (EMG) instrumentation is to accurately record and display the nerve and muscle signals that are physiologically relevant. Another way of expressing the same goal is that the EMG machine must discard everything that is not physiologically relevant-it must throw out $99 \%$ of the total signal. Understanding EMG electronics is important because small changes in an instrument can produce large alterations in the display, particularly when the in-

From the Department of Physical Medicine and Rehabilitation, University of Michigan Medical Center, Ann Arbor, Michigan.

Acknowledgments: This work was supported by Grant NS-01701 from the National Institutes of Health. An earlier version of this material was presented as part of the Course B Nerve Conduction Studies at the 1988 annual AAEM meeting, San Diego, CA. I thank L. Perry for secretarial assistance.

Address reprint requests to American Association of Electrodiagnostic Medicine (formerly the American Association of Electromyography and Electrodiagnosis), 21 Second Street S.W., Suite 306, Rochester, MN 55902

Accepted for publication November 4, 1990

CCC 0148-639x/91/0100937-010 $\$ 04.00$

(c) 1990 Daniel T. Barry, MD, PhD

Published by John Wiley \& Sons, Inc strument is discarding such a large proportion of the original signal.

The topics in this monograph are discussed at an introductory level to provide understanding for readers with no electronics background, and intuitive insight for more experienced readers. Basic principles of electricity include the concepts of voltage, current, charge, and impedance that allow discussion of filters, amplifiers, electrodes, digital electronics, stimulators, and patient safety. The appendix provides an intuitive introduction to voltage, current, and impedance.

\section{ELECTRICITY}

The basis of electricity is charge and charge flow. Charge is a concept that was originated to explain experimental results. Benjamin Franklin performed the experiment of rubbing a glass rod with silk and noted that the rod subsequently attracted the silk. ${ }^{6}$ If a second glass rod is rubbed with silk, the two rods repel each other. Presumably, two substances that were initially balanced in both the glass and the rod were somehow separated, so that more of one ended up on the glass and more of the other ended up on the silk. Franklin arbitrarily named the charge on the glass rod positive and the charge on the silk negative. The electric field was invented to explain the force between the two objects.

The movement of charges corresponds to current flowing from one object to the other. The 
current (amperes or amps) is defined as the amount of charge (coulombs) flowing per unit of time (seconds). Positive charges flowing from silk to rod are added to negative charges flowing in the opposite direction, from rod to silk, to determine the total current flow. Current has a direction, and the direction could be defined as either the net rate of flow of negative charges or the net rate of flow of positive charges. The arbitrary convention is that current is the net rate of flow of positive charges. This convention is why diagrams are drawn showing current flowing from positive $(+)$ leads to negative $(-)$ leads. The fact that the usual charge carriers are negative (electrons), and that flow is in the opposite direction to the one indicated, can cause confusion, but is irrelevant for circuit design. In the rod-silk example, the convention implies that current flowed from silk to rod. Physical reality is that electrons flowed from rod to silk.

The three parameters that form the basis of instrument design are voltage, current, and impedance. Voltage (V) corresponds to potential energy per unit of charge (joules/coulomb), current (I) is charge flow (coulombs/second), and impedance (Z) is, for most signals, simply the ratio of voltage to current (ohms) (Equation 1). An intuitive approach to understanding these parameters is presented in Appendix A.

$$
\mathrm{V}=\mathrm{I} * \mathrm{Z}
$$

Equation 1 implies that if two of the three parameters are known, then the third can be calculated. A simple measurement of current is obtained by making an electromagnet. Current flowing through a wire produces a magnetic field. The current can be measured by determining the strength of its magnetic field, e.g., by measuring the force exerted on a piece of iron. Voltage between two locations can be measured by measuring the force on a charged object when the object is placed at one of the locations and comparing that force to the force measured when the charged object is placed at the other location. A simpler method utilizes equation 1 . The two locations are connected with a known impedance, and the resulting current's magnetic field is used to deflect a piece of iron (Fig. 1).

Measuring a fixed current flow or direct current (DC) provides a straightforward answer (e.g., $10 \mathrm{~A}$ ). How does one describe current flow that varies with time? Describing the entire waveform

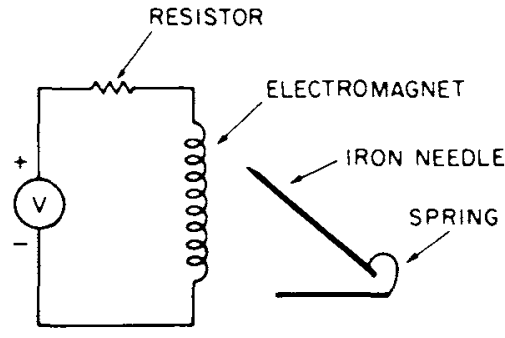

FIGURE 1. The magnetic field created by current flow can be used to deflect an iron needle and indicate the amount of current that is flowing. The stronger the current, the greater the needle deflects.

is cumbersome, and just reporting the peak-topeak amplitude is misleading in the cases of currents containing occasional, short-duration, large transients. A convenient measure of current flow or of voltage is the root-mean-squared (RMS) value of the waveform (Equation 2). The RMS value of current is the value displayed by almost all alternating current (AC) voltmeters. This complicated-looking value is useful because it is a measure of the power contained in the signal. For sine waves, the RMS value is simply 0.707 times the amplitude of the sine wave. A familiar example is the wall voltage of $120-\mathrm{V}$ RMS - the amplitude of the voltage is actually $170 \mathrm{~V}(120 / 0.707)$ and the peak-to-peak amplitude is $340 \mathrm{~V}$.

$$
I_{\mathrm{rms}}=\sqrt{\frac{1}{T} \int_{0}^{T}(i(t))^{2} d t}
$$

where $t=$ time, $T=$ total time the signal exists, $i(t)$ $=$ current at time $t$, and $I_{\mathrm{rms}}=$ root mean squared current.

Descriptions of electric signals frequently refer to the frequency content of the waveform. These descriptions rely on the fact that any arbitrary waveform can be represented as a sum of sine waves of various frequencies, amplitudes, and phases (Fig. 2). If very high-frequency sine waves are needed to obtain a good match between the waveform and the sum of sine waves, then the waveform has high-frequency components. Such a waveform is characterized by rapid fluctuations in time; the rapid fluctuations correspond to the high-frequency components. High-frequency potentials decrease in amplitude with distance from muscle fibers much more than low-frequency potentials. Tissue acts as a low-pass filter, i.e., selectively removes the high-frequency components of the signal. ${ }^{2}$ This explains the familiar finding that motor units recorded from far away appear 


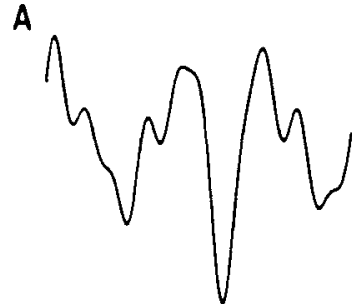

B

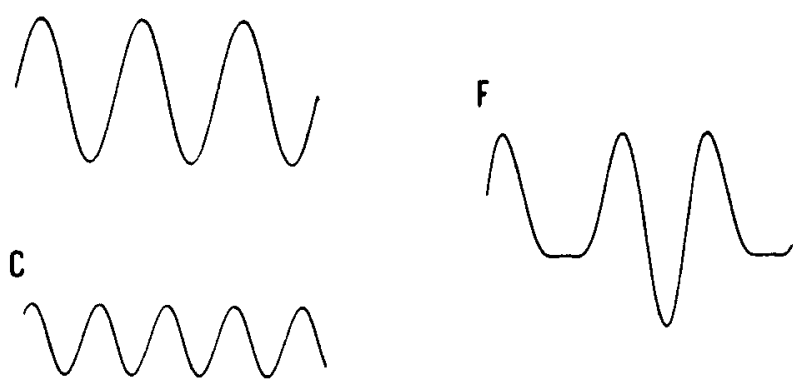

G

D

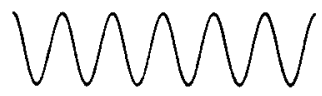

E
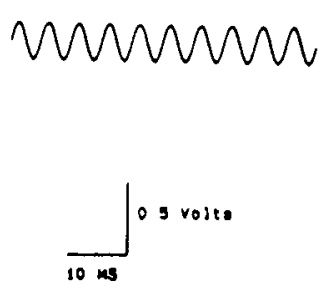

FIGURE 2. Any waveform can be represented as a sum of sine waves. (A) Waveform to be represented. (B) Sine wave: $60 \mathrm{~Hz}$, 0 phase, 1 V. (C) Sine wave: $90 \mathrm{~Hz}, 45^{\circ}$ phase, $0.5 \mathrm{~V}$. (D) Sine wave: $120 \mathrm{~Hz}, 90^{\circ}$ phase, $0.5 \mathrm{~V}$. (E) Sine wave: $200 \mathrm{~Hz}, 0$ phase, $0.25 \mathrm{~V}$. (F) Sum of (B) and (C). (G) Sum of $B, C$, and $D$. (H) sum of $(B),(C),(D)$, and $(E)$.

smoother and of lower amplitude than when the needle is right next to muscle fibers in the motor unit.

\section{FILTERS}

Every device that conducts an electric signal changes the signal, i.e., filters it. Most devices are designed to minimize their filtering effect. An example is the power cord connected to the wall; quality power cords are made with generous amounts of copper wire to minimize the resistance of the cord. Design criteria such as cost, size, safety, and convenience often result in compromise, allowing filtering to become significant. Recording routine conduction studies with surface electrodes instead of needles is an example of appropriate compromise. Also, EMG needles and amplifiers have filtering effects that reflect design compromises.

Some devices are designed to perform specific filtering actions. Examples are the low-pass and high-pass filters on EMG machines. The high-pass filter is the one typically set to a low frequency, e.g., $20 \mathrm{~Hz}$, since it allows high frequencies through and stops low frequencies. For this reason, the high-pass filter is sometimes called the low-linear frequency filter, while the low-pass filter is sometimes called the high-linear frequency filter-a confusing set of terms. The purpose of these devices is to eliminate the frequency components of a signal that corresponds to noise while preserving the frequency components that correspond to the actual physiologic signal. Figure 5 shows the effect of introducing high- and low-pass filters inappropriately.

Most filters are designed with capacitors and resistors. Capacitors are devices that impede current flow by an amount that depends on the frequency content of the current. Capacitors completely block direct current (DC or zero frequency) signals, completely pass signals of infinite frequency, and partially impede signals of finite frequency, the impedance decreasing as frequency increases (Equation 3).

$$
\left|Z_{\text {cap }}\right|=\frac{1}{2 \pi f C}
$$

where $\left|Z_{\text {cap }}\right|=$ magnitude of the capacitive impedance, $f=$ frequency, and $C=$ capacitance.

Think of capacitors as valves that only let high frequencies through. A high-pass filter is created by placing a capacitor in the signal path. Only high-frequency components get through. A lowpass filter is created by connecting a capacitor between the signal path and ground. The high-frequency components leave the signal path and go to ground, leaving only the low-frequency components to travel further down the path. Capacitors also change the phase of a signal. A sine wave is shifted by $90^{\circ}$ in phase when it passes through a capacitor. Resistors provide a constant impedance to current flow, regardless of frequency, and do 
not produce any phase shift. The combination of resistance and capacitance determines the cutoff frequency of the filter (Equation 4).

$$
f_{\mathrm{co}}=\frac{1}{2 \pi R C}
$$

where $f_{\text {co }}=$ cutoff frequency, $R=$ resistance, and $C=$ capacitance.

The cutoff frequency is the frequency at which the output signal amplitude is only about $70 \%$ (actually $(1 / 2)^{1 / 2}$ ) of the input signal amplitude. The cutoff frequency is also the frequency at which half of the maximal phase shift occurs. Note that setting a 2-kHz low-pass filter does not imply that all frequency components below $2 \mathrm{kHz}$ pass through unscathed. There is a smooth transition from frequencies mostly passed to frequencies mostly blocked, with $2-\mathrm{kHz}$ frequency components being reduced to $70 \%$ of their input amplitude. The abruptness or "sharpness" of the cutoff varies with different designs. Often, a compromise is made between the sharpness of the cutoff and distortion from phase changes produced by the filter. The effect is similar to phase distortion in an amplifier (Fig. 5D).

Digital filters provide a simple technique for optimizing filter performance under varying conditions without expensive hardware. To change filters, one simply runs a different computer program rather than physically switching to different hardware components.

\section{INPUT IMPEDANCE}

Devices are frequently joined in series so that the output of one is the input to the next (Fig. 3). Each device can be considered independently as a filter, or "system," with certain input/output characteristics. The input/output characteristics of the group are determined entirely by combining only the input/output characteristics of each device, without regard to the internal workings or structure of the device. This approach works for simple devices, such as resistors, and for more complicated devices, such as amplifiers or computers.

Connecting resistances in series produces a device with resistance equal to the sum of the individual resistors (Equation 5).

$$
R_{\mathrm{tot}}=\mathrm{R}_{1}+\mathrm{R}_{2}
$$

where $R_{\text {tot }}=$ total resistance, and $\mathrm{R}_{1}, R_{2}=$ individual resistances.

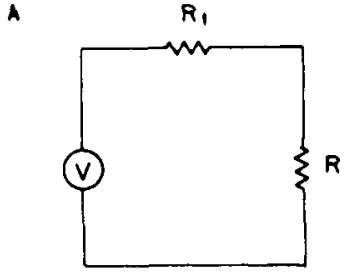

日

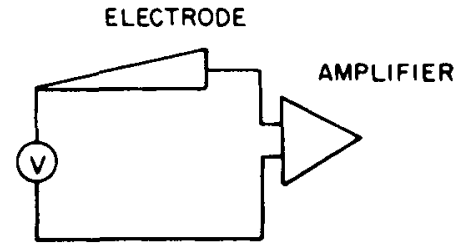

FIGURE 3. Impedances connected in series are summed to determine the total impedance. (A) Resistors in series. The total voltage is divided between the two resistors according to equation (6). (B) An electrode and amplifier in series. The voltage across the amplifier will be near the voltage in the muscle only if the amplifier input impedance is much higher than the electrode impedance.

For a given voltage, the current that will run through the device can be calculated using equation (1). Since there is only one pathway in the device, the same current will go through each resistor. Knowing the current passing through the whole device allows calculation of the voltage that appears across individual resistors (Fig. 3, Equation 6).

$$
\mathrm{V}_{R}=\frac{R}{R_{1}+\mathrm{R}} V
$$

Note that equation 6 is easily derived from equations 1 and 5 . A consistency check is made by adding the voltages across individual resistors; the sum must equal the total voltage across the device. For example, if there are only two resistors in the circuit, and they have the same resistance value, then the voltage across each resistor must be half the total voltage applied to the circuit.

Series resistors provide an illustration of the benefit of high-input impedance. The input impedance of a device is a measure of how much current flow is needed to produce a particular voltage (see Equation 1) across the input terminals of the device. Consider the case of an electrode attached in series to an amplifier (Fig. 3B). The electrode is placed in a muscle and a voltage is imposed between the active electrode and the reference. The current that flows is determined by the sum of the electrode and amplifier impedances. The sum of the voltage across the electrode 
and the voltage across the amplifier will equal the total voltage imposed between the active electrode and reference. As illustrated in Figure 3B, if the electrode impedance is high, then most of the voltage will drop across the electrode, leaving little to drop across the amplifier. Only the voltage that drops across the amplifier, however, can be detected by our apparatus and go on to be recorded and displayed. If the amplifier is to detect $99 \%$ of the voltage appearing at the electrode, then the amplifier input impedance must be 99 times greater than the electrode impedance. This is why an EMG amplifier must have an input impedance that is much higher than the needle electrode impedance.

\section{AMPLIFIERS}

An ideal amplifier converts a low-voltage waveform to a higher-voltage copy of the same waveform. To accomplish such a transformation, the ideal amplifier has an infinite input impedance, infinite frequency response, introduces no noise, is perfectly linear, and has an infinite dynamic range. Real amplifiers, with finite parameters, filter the signal as they amplify it. A good design only minimally filters waveforms encountered in normal operation. One can think of a real amplifier as an ideal amplifier coupled to filters that are permanently set to reflect the actual performance of the amplifier (Fig. 4).

The dimensionless ratio of the output voltage to the input voltage is the gain of the amplifier. Amplifier sensitivity relates the input voltage to a display scale, e.g., $1 \mathrm{mV} / \mathrm{cm}$. If the amplifier sensitivity is too low, then small deviations from baseline will be unapparent, resulting in artifactual longer latencies to takeoff in nerve conduction studies, and apparent decreased duration of motor unit action potentials. ${ }^{8}$

An inadequate amplifier will distort the wave-

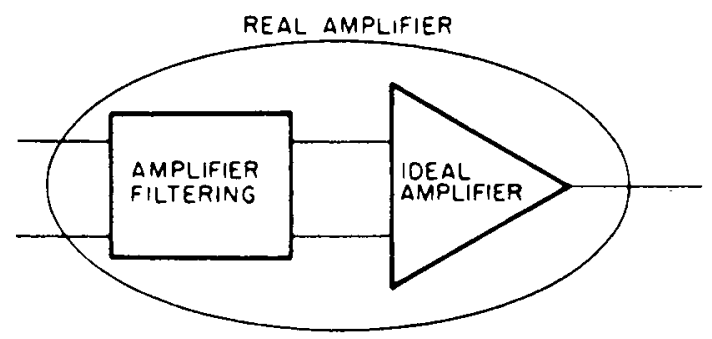

FIGURE 4. A real amplifier can be represented as an ideal amplifier that is permanently connected to filters that reflect the limitations of the real amplifier.
A
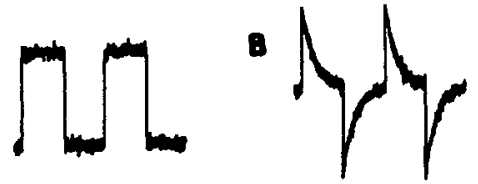

C

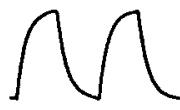

1.0 Volt
D1

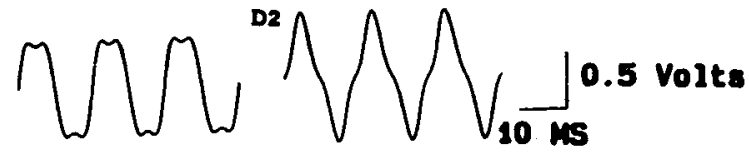

FIGURE 5. Filters distort signals in characteristic ways. (A) A square wave with superimposed noise. (B) High-pass filtering results in decay of steady potentials. The flat parts of the square waves are distorted. Extra phases can be introduced. (C) Lowpass filtering causes high-frequency components to be lost. The result is reflected in reduced amplitudes and smoother waveforms as rapid, high-frequency deflections are eliminated. Note the reduction in rise time of the square wave signal. (D) 1 . This signal consists of a $1.0-\mathrm{V}, 60-\mathrm{Hz}$ sine wave added to a $0.2-\mathrm{V}$, $120-\mathrm{Hz}$ sine wave. 2 . Simply changing the phase of the $120-\mathrm{Hz}$ wave produces a dramatic distortion of the signal. Here the $120-\mathrm{Hz}$ wave is shifted by $180^{\circ}$ before adding it to the $60-\mathrm{Hz}$ sine wave. Phase distortion is the result.

form. If the amplifier's frequency response is limited, then high- or low-frequency components may be lost (Figs. 5A, 5B, and 5C). Frequencies near the cutoff frequency can produce distortion due to a poor phase response (Fig. 5D). If the amplifier's gain varies with the level of input voltage, then the amplifier is nonlinear and can produce bizarre distortions. A familiar nonlinearity occurs when the input voltage is too large, and the output saturates or blocks.

When a small signal is embedded in environmental electric noise, two amplifiers can be combined to create a differential amplifier that will dramatically improve the signal-to-noise ratio (Fig. 6). The "trick" is to place the input leads of the two amplifiers very close to each other so that the environmental noise will be the same on both leads. Then the leads are attached so that the desired signal will appear as a voltage between the two leads. Ideally, the leads will carry the same noise, but only the active lead will carry the signal. The differential amplifier will invert and amplify the signal from one lead (reference lead), and 
then add it to the amplified signal from the other lead (active lead). If the two amplifiers are identical, then the noise, common to both leads, will cancel but the signal will be preserved. If the amplifiers are not identical, then one will amplify the noise more than the other and the sum of the two signals will no longer completely cancel.

The common mode rejection ratio (CMRR) is a measure of how identical two amplifiers are (Fig. 6). As an example, suppose the amplifiers are not identical; let amplifier 1 have a gain of 100 , and amplifier 2 have a gain of 99.9 . A $2.0-\mathrm{V}$ signal common to both inputs will result in $200 \mathrm{~V}$ from amplifier 1 and negative $199.6 \mathrm{~V}$ from amplifier 2 . Summing the two voltages yields an output of 0.4 $\mathrm{V}$ from the differential amplifier. The common mode gain is the ratio of the output to the common input voltage. In this example, the common input is $2.0 \mathrm{~V}$ and the output is $0.4 \mathrm{~V}$, so the common mode gain is 0.2 . If $2.0 \mathrm{~V}$ had been applied differentially, perhaps as $+1 \mathrm{~V}$ to amplifier 1 and $-1 \mathrm{~V}$ to amplifier 2, then the output would be $200 \mathrm{~V}+$ $196 \mathrm{~V}$, or $396 \mathrm{~V}$, for a "differential gain" of 396 $\mathrm{V} / 2 \mathrm{~V}$ or 198 . The CMRR is the ratio of differential gain to common mode gain, in this case, 198/ 0.2 , or 960 . Expressed in decibels [20 $\log _{10}$ (ratio)], a ratio of 960 is $59.6 \mathrm{~dB}$.

\section{ELECTRODES}

Three electrodes are always attached to the patient during nerve conduction studies (NCS) and EMG: an active electrode, a reference electrode, and a ground electrode. As discussed above, the displayed signal will be the voltage difference between the active and reference electrodes. The

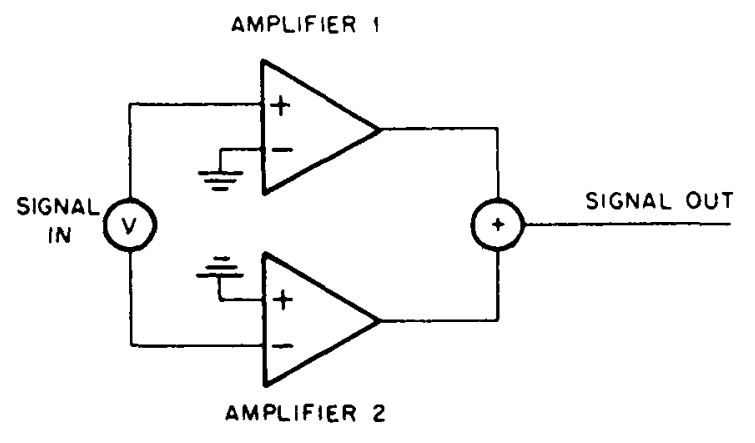

DIFFERENTIAL AMPLIFIER

FIGURE 6. A differential amplifier is created by combining two amplifiers. One signal is inverted, and the outputs of the amplifiers are added. Common mode rejection is a measure of how well the differential amplifier eliminates signals that are present on both inputs (see text). ground electrode serves as a safety feature and a low impedance path to drain electric noise away from the signal electrodes.

For sensory NCSs, the two signal electrodes are both placed over the nerve or around a finger, about $4 \mathrm{~cm}$ apart. The $4-\mathrm{cm}$ distance is appropriate because a typical action potential duration of $0.8 \mathrm{~ms}$ and a typical velocity of $50 \mathrm{~m} / \mathrm{s}$ produces a depolarization that extends for $4 \mathrm{~cm}(500 \mathrm{~cm} / \mathrm{s} \times$ 0.008 seconds). Note that the differential recording produces a biphasic signal when a positive, monophasic signal appears first at the active lead and then at the reference lead. If the leads are too close together, the signal may be distorted as the beginning of the signal appears in the reference lead before the end of the signal has passed the active lead. Motor NCSs use electrodes placed over the muscle motor point and a tendon. The compound muscle action potential (CMAP) is large enough so that some of the signal will generally appear in the reference electrode.

Needle electrodes record extracellular voltages in muscle. The voltages reflect membrane current as it flows through extracellular fluid. Recall that the current flows in a loop across the muscle fiber membrane into the extracellular fluid, through the extracellular fluid longitudinally along the axon, back across the muscle fiber membrane into the cell, and through the cytoplasm back to the starting point. Note that voltages across the membrane, resting potentials, and action potentials, are not measured by the electrode. The electrode records the voltage between two extracellular locations; this voltage depends on membrane current, not membrane voltage.

The metal of a needle electrode interacts with the extracellular fluid to create a charge separation similar to Franklin's glass and silk. The resulting voltage is called a tip potential or polarization potential. These potentials can be large but generally are not noticed since they are DC signals and the amplifiers are AC coupled. Dirt or frayed Teflon, however, can cause the tip potentials to be unstable and can produce artifacts that can be mistaken for spontaneous muscle fiber activity.

The two most common EMG electrodes are the monopolar and concentric electrodes. The monopolar electrode is combined with a reference electrode placed subdermally nearby. Monopolar electrodes have much greater separation between active and reference sites than concentric needles. The increased separation increases noise and motor unit action potential (MUAP) size compared to concentric needle recordings. If the Teflon tip 
frays, more of the needle is used for recording, the impedance drops, and the signal is averaged over a wider volume of muscle. The result is a reduction in amplitude and polyphasia of MUAPs that can seriously distort the waveform. ${ }^{3}$ Electrodes have capacitance so the electrode impedance decreases with increasing frequency. Weich$\mathrm{ers}^{9}$ demonstrated an average impedance of monopolar electrodes of $1.4 \mathrm{M} \Omega$ at $10 \mathrm{~Hz}$, compared with $4.7 \mathrm{M} \Omega$ for concentric electrodes at 10 $\mathrm{Hz}$. Thin concentric needles $(<0.03$ square millimeter area) may have high enough impedances to compromise the performance of amplifiers that have adequate input impedances for monopolar and larger concentric needles. Although concentric needles do not have the problem of Teflon fraying, there is considerable variation in electrode characteristics among manufacturers. ${ }^{5}$

\section{DIGITAL ELECTRONICS}

The essence of digital storage is that any waveform can be characterized by a list of numbers. The waveform and the number list are completely equivalent; however, computers are confined to working with lists of numbers while humans have the capability to visualize and understand information presented as a waveform. The process of converting a waveform to a number list is called analog-to-digital or A-to-D conversion. The reconstruction of the waveform from numbers is D-to-A conversion.

Number list representations are frequently used as a simple method of saving the waveform for later inspection. For example, without storage, the CMAP appears as a quick transient event on the oscilloscope screen. By storing the CMAP as a list of numbers, it can be repeatedly displayed, providing the visual impression of a fixed image.

The details of digitization determine the quality and accuracy of the reconstructed and displayed waveform. If the digitization rate is too slow, then the time between two numbers on the list will be too long. The result is poor resolution in time, so that sharp spikes appear blunted or may be missed entirely. Since time is displayed along the horizontal axis, slow digitization rates produce poor horizontal resolution. Inadequate horizontal resolution prevents most digital signal processing from working properly. Poor vertical resolution occurs when the range of numbers allowed is too small. For example, if the numbers are restricted to $0,1,2$, or 3 , then each integer covers $25 \%$ of the entire range of vertical resolu- tion. The reconstructed waveform will have only four possible voltages and will look choppy, like a staircase with high vertical steps. Since numbers in a computer are always represented in binary format, the range of allowed numbers is expressed in bits. For example, the four integers from 0 to 3 represent 2-bit resolution $(00,01,10,11)$. Twelvebit resolution allows $2^{12}$ or 4096 possible numbers (000000000000, 000000000001, 000000000010 , $000000000011, \quad 000000000100$,

111111111111 ), so that each number covers only $0.02 \%$ of the entire range.

The number list can be used to determine amplitude between two points by subtracting the number corresponding to one point of the waveform from the number corresponding to another point. Averaging is used to improve the signal-tonoise ratio. Averaging is accomplished by combining several number lists; the lists are added and the sum is divided by the number of lists to produce a single, averaged list. Similarly, all of the analog operations of filtering can be accomplished through appropriate manipulation of the number list.

Delay lines are made by continually updating a number list that represents the most recent waveforms recorded. The delay line allows inspection of events that occurred prior to a particular event of interest. For example, when isolating a MUAP, one usually triggers on the portion of the MUAP near the peak. Without a delay line, inspection of the MUAP waveform is limited to the portion occurring at and after the time the trigger is activated. The delay line contains data that occurred just before the trigger was activated, allowing inspection of the entire MUAP even if triggering occurs at the peak.

Digital representation of waveforms allows signal processing techniques to be used to interpret the waveforms. Computer programs can automatically determine latency and amplitude values of evoked potentials. Many algorithms exist to help interpret interference patterns and MUAP characteristics, such as turns and amplitude ratios. ${ }^{4,7}$

\section{STIMULATORS}

Stimulators are used to induce action potentials in nerve fibers. Current flows from the anode (+ pole) of the stimulator to the cathode ( - pole). Some current will flow inside each axon, from the site under the anode toward the site under the cathode. With the membrane at rest, only a few charges actually flow across the membrane, and 
the membrane potential is linked to membrane current flow primarily through the capacitive properties of the membrane. Positive charges accumulating inside the axon, under the cathode, cause a depolarization as the capacitive charge is reduced (positive charges accumulating on the inside cancel the negative charges that are sustaining the membrane potential).

Current also flows just outside the axon from the anodal region to the cathodal region. Why don't these two current transfer the same amount of charge, both inside and outside the axon, so that the net effect is zero? The difference is that the charges inside the axon are confined, but the charges outside the axon can flow toward or away from the stimulator. Positive charges outside the axon flow toward the axon under the anode, replacing the charges that flowed toward the cathode. Positive charges outside the axon flow away from the axon under the cathode, draining the charges that flowed away from the anode. The net result of all these current flows appears as a current that is flowing out into the axon under the anode and out of the axon under the cathode, resulting in depolarization of the axon under the cathode. The initial depolarization opens regenerative channels, sodium ions flow in, and an action potential is created under the cathode. The action potential is capable of propagating in both directions of the axon; however, the part of the nerve under the anode is hyperpolarized, so the action potential may fail to propagate past the anode (anodal block).

Myelinated axons are easier to stimulate than unmyelinated axons because essentially all the stimulus current exits the axon at the tiny nodes of Ranvier. Since the current flows out of a small area of membrane, the current is very concentrated and small currents produce significant local depolarization. Large axons are easier to stimulate than small axons for a similar reason. As the radius of an axon increases, the ratio of membrane area to cross-sectional area decreases. Also, the current flow produced by the stimulator in an axon is proportional to the cross-sectional area of the axon. Therefore, the stimulus current flowing through the membrane of a large axon will be more concentrated than in smaller axons.

Stimulators can provide either constant current or constant voltage stimulation. The constant voltage mode creates a voltage across the anode and cathode; the voltage is determined by a dial setting. Current flow is determined by the impedance of the skin, fluid, and tissues between the two poles. If the poles are held in the open air, essentially no current flows. If the poles are connected by a good conductor, such as a wire, then the current is only limited by safety features in the machine (i.e., buy a new fuse). Constant current stimulators provide a specific current that is selected by a dial setting. In this case, the voltage across the poles is determined by the impedance between the poles. If the poles are connected by a wire, the selected amount of current flows (with very little voltage across the poles). If the poles are held in the open air, then the maximum voltage allowed by the machine will appear across the poles and, in a good design, an indicator will inform the operator that the impedance limit was exceeded and the desired current did not flow. Constant current stimulators are much more useful than constant voltage stimulators in determining thresholds to stimulation. Variations in skin impedance alone will produce apparent threshold changes when using constant voltage stimulators.

Stimulators can develop large internal charges and voltages. Also, the discharge of a stimulator can introduce transients into other equipment, producing artifacts and, sometimes, damage. Stimulus isolation units typically use transformers or optical circuits to isolate the patient and other equipment from the stimulator circuitry.

\section{PATIENT SAFETY}

Where there is voltage, there is current, i.e., there is no such thing as an infinite impedance. Stray currents from apparatus are termed leakage currents and patients must be protected from them (Table 1). Electric currents can be induced in materials that are simply a nearby power source. Current flows from a metal chassis to ground because voltages are created on the chassis from power cords and transformers inside or near the chassis. Ground receptacles are not all at the same voltage so current will flow between ground leads of devices that happen to be plugged into different wall sockets. Extension cords should not be used since they increase the differences between receptacles and can induce unacceptable levels of current.

Precautions include using only a single grounding point on the patient and tying all equipment grounds to a common place that is grounded. Patients must be kept away from ungrounded surfaces or devices that could produce excessive currents when the EMG electrodes are attached. Examples include an electric bed with a metal frame and broken ground lead or a lamp with a two-prong power plug. 
Table 1. Summary of risk current requirements in RMS microamperes (DC to $1 \mathrm{kHz}$ ).

\begin{tabular}{l}
\hline \\
\cline { 5 - 7 }
\end{tabular}

Very small electric currents are capable of inducing ventricular fibrillation, particularly if the skin resistance is eliminated by a needle. The American National Standards Institute (ANSI) approves test methods and safety standards ${ }^{1}$ that set limits on current flow for electromedical apparatus used in patient areas (Table 1).

The highest safety category is termed "isolated" patient connection. Isolated equipment is used in situations where the skin is not intact, so a pathway can conduct current to the heart. Isolated conditions require the equipment to generate less than $10 \mu \mathrm{A}$ RMS from contact leads to ground (source current). It must also not allow more than $10 \mu \mathrm{A}$ (sink current) to flow through the device to ground if patient leads come in contact with a $120-\mathrm{V}, 60-\mathrm{Hz}$ source. The sink current requirement helps to protect a patient who is inadvertently exposed to the wall voltage while being attached to an electromedical device. Up to $100 \mu \mathrm{A}$ of current is allowed between the chassis and ground, tested both with the ground intact and with the ground disconnected.

The use of electrosurgical units (ESUs) for cutting and coagulating during surgery creates a very high-frequency electric field that can result in substantal current flow if devices are not adequately protected. The very high frequencies of ESUs can cause damage to solid state devices and can bypass standard safety measures designed to protect against $60-\mathrm{Hz}$ risks. For example, the cables used as patient leads may have very low impedances at the frequencies used by ESUs. Some ESUs provide digital outputs to communicate to other devices when the ESU is activated, and the devices can be designed to disconnect during ESU operation.

\section{SUMMARY}

Electrodiagnostic instrumentation is designed to accurately measure and display tiny physiologic signals. The straightforward principles of voltage, current, and impedance provide an adequate base to understand design criteria such as wideband frequency responses, variable gains, high-input impedances, good signal-to-noise ratios, and large common mode rejection ratios. The accuracy and interpretation of test results, the selection of appropriate equipment for purchase, and patient safety are all enhanced by an understanding of fundamental electronics. Furthermore, these principles provide insight into the physiologic events being measured in electrodiagnostic studies.

\section{APPENDIX: INTUITIVE APPROACH TO ELECTRIC PARAMETERS}

The concepts of voltage, current, and impedance are easily related to more intuitive parameters, allowing basic understanding to grow from a conceptual basis as well as from quantitative analysis. Mechanical systems, fluid fow, heat transfer, and electric systems are all described by the same differential equations. To understand any one of these processes is equivalent to understanding them all. The only difference is the parameters. Substituting voltage for pressure and electric current for fluid flow allows an electric engineer to work in fluid mechanics. 
Voltage is a measure of electric potential energy just as height is a measure of gravitational potential energy in the approximately constant gravitational field near the earth's surface. The gravitational potential energy of an apple is realized when it drops from the tree. The apple's velocity, when it hits your head, depends on the difference between the original height of the apple and the height of your head. Both height and voltage are measured as a difference between two locations, rather than as absolute numbers. Note that height is frequently expressed as an absolute value such as the height of Mt. Everest, or an individual's height. The reference height could be general, such as mean sea level, or local (e.g., the ground the individual is standing upon). Absolute voltage values (e.g., $120 \mathrm{~V}$ ) are frequently referenced to a metal post buried in the ground near the power transformer outside the building. Test equipment, however, measures voltages with respect to a very local reference such as the ground receptacle in a wall plug. One should be aware that there are frequently measurable voltages between the different ground receptacles in a single room.

Current is a measure of flow. A river current corresponds to the volume of water that flows in some amount of time (e.g., liters per second). The current is determined by the steepness of the river grade ("voltage") and the friction of the water and river bed ("resistance"). A wide river flowing from a steep mountain passes huge amounts of water, just as a small resistor and a large voltage results in a huge electric current.

For direct currents (DC), impedance is the same as resistance, and simply corresponds to friction in a mechanical system. When alternating currents (AC) are used, however, some of the energy from one cycle can be stored for use in later cycles. This concept of energy storage forms the basis of impedance. A classic example of mechanical impedance is the mass-spring-friction system. If friction is eliminated, starting the mass and spring bouncing results in their bouncing forever. This is impedance without resistance. Energy is simply shifted back and forth between the mass and spring. For a while, the moving mass (kinetic energy) compresses the spring (potential energy), then, for a while, the compressed spring moves the mass. The frequency of bouncing is termed the resonant frequency of the mass-spring system and is determined by the spring constant and the mass. If friction is introduced, then energy is dissipated as heat and, over time, the bouncing subsides. Similarly, an ideal capacitor and inductor will pass electric energy back and forth forever oscillating between energy storage in an electric field (capacitor) and a magnetic field (inductor). Including a resistor causes the oscillations to die away as energy is dissipated as heat.

\section{REFERENCES}

1. ANSI/AAMI American National Standard: Safe Current Limits for Electromedical Apparatus, ES1-1985. Arlington, VA, Association for the Advancement of Medical Instrumentation, 1985 .

2. Butler BP, Ball RD, Albers JW: Effect of electrode type and position on motor unit action potential configuration. Muscle Nerve 1982;5:S95-S97.

3. Chu J, Chan R, Pruyninckx F: Progressive Teflon denudation of the monopolar needle: Effects on motor unit parameters. Arch Phys Med Rehab 1987;68:36-40.

4. Desmedt JE (ed): Computer-Aided Electromyography. Basel, Karger, 1983.

5. Dorfman LJ, McGill KC, Cummins KL: Electrical properties of commercial concentric EMG electrodes. Muscle Nerve $1985 ; 8: 1-8$
6. Halliday D, Resnick R: Fundamentals of Physics. New York, John Wiley \& Sons, 1974, p 423.

7. Nandedkar SD, Sanders DB, Stålberg EV: Automatic analysis of the electromyographic interference pattern in normal muscle. Part 1: Turns and amplitude analysis. Muscle Nerve 1986;9:423-430.

8. Stolov WC: AAEM Minimonograph \#16: Instrumentation and Measurement in Electrodiagnosis. Rochester, MN, American Association of Electrodiagnostic Medicine, formerly American Association of Electromyography and Electrodiagnosis, 1981.

9. Weichers DO: EMG needle electrodes. Arch Phys Med Rehabil $1979 ; 60: 394$. 